\title{
Spinal Dermal Sinus and Sacral Endothelial Cyst with Recurrent Bacterial Meningitis: A Case Report and Systematic Review of the Literature
}

\author{
Dan Yu ${ }^{1}$, Meng Mao', Hui Zhou ${ }^{1}$ and Liang Huang ${ }^{2,3 *}$ \\ ${ }^{1}$ Department of Pediatrics, West China Second University Hospital, Sichuan University, China \\ ${ }^{2}$ Department of Pharmacy, West China Second University Hospital, Sichuan University, China \\ ${ }^{3}$ Key Laboratory of Birth Defects and Related Diseases of Women and Children, West China Second University Hospital, Sichuan University, China
}

Received: December 21, 2017; Published: January 05, 2018

*Corresponding author: Liang Huang, Department of Pharmacy/Evidence-Based Pharmacy Center, West China Second University Hospital, Sichuan University, Chengdu 610041, China; Email: kaoliao1@163.com

Abbreviations: CRP: C-Reactive Protein; CT: Computed Tomography; CSF: Cerebro-Spinal Fluid; ESBL: Extended spectrum beta-lactamases; MRI: Magnetic resonance imaging

\section{Introduction}

Congenital dermal sinus is dermoid and epithelial cyst from skin tissue or epithelial tissue residue in the cranial cavity or intraspinal canal during embryonic development. The entry of sinus is usually located in the occipital, back, waist midline skin, with hair hyperplasia, and sinus connect skin with nerve tissue which constitute the anatomical basis of infection of the nervous system. We report 1 case of recurrent bacterial meningitis caused by sacral sinus and sacral canal endothelial cysts. Meanwhile, the related literatures are systematically searched and reviewed, which provide experience for the diagnosis and treatment of bacterial meningitis caused by congenital sinus.

\section{Case Presentation}

A previously healthy 8 month-old boy was admitted because of a 9 days' fever and a 2 times' convulsions. Physical examination revealed a febrile, obtunded with anterior bulging fontanel. Neck stiffness was noted. Laboratory tests showed WBC10.6 $\times 10^{9} / \mathrm{L}$, neutrophile granulocyte $58.5 \%$. C-reactive protein (CRP) $93 \mathrm{mg} / \mathrm{L}$ (normal value below $8 \mathrm{mg} / \mathrm{L}$ ). An urgent computed tomography (CT) of the head was performed, with normal results. Bacterial meningitis was suspected and a lumbar puncture confirmed the diagnosis. The cerebro-spinal fluid (CSF) was $8200 \times 10^{6} / \mathrm{L}$ white blood cells with neutrophile granulocyte $88 \%$, increased total protein $(190,7 \mathrm{mg} / \mathrm{L})$ and decreased glu-cose levels $0.2 \mathrm{mmol} / \mathrm{L}$. Serum glucose level was normal $(5 \mathrm{mmol} / \mathrm{L})$. The child received intravenous dexamethasone and ceftriaxone $(100 \mathrm{mg} / \mathrm{kg}$. d) as empiric treatment which results of poor effects, so vancomycin $(60 \mathrm{mg} / \mathrm{kg}$. d) was added. Escherichia coli with ESBL (+) was confirmed in both double blood and cerebrospinal fluid (CSF) cultures, which was sensitive to carbapenems and amino glycosides. So we replaced ceftriaxone with meropenem $(120 \mathrm{mg} / \mathrm{kg}$. d) in treatment.

Five days later, the child's condition improved, with fever resolution. Suddenly, 10 days after hospital admission, the child manifested irritability and lethargy. Moreover, a bulging fontanel was noted at the physical examination. During the hospitalization time (51 days) of the child, we used cefatriaxone (4days), vancomycin (21days), meropenem (47days), compound sulfamethoxazole (60mg/kg.d) (7days, combined with meropenem), amikacin15mg/ kg.d (15days, combined with meropenem). We noticed that after the condition and examinations of child had been improved for several days, it would become worsen again. This had happened several times. Then, the patient transferred to another hospital. A needle sized skin dimpling was noticed at sacrococcygeal this time. And the child's parents told the doctor that sacrococcygeal sinus was found after the child was born. Brain MRI revealed ventricular dilatation and hydrocephalus. Lumbar vertebra MRI revealed sacral sinus, extra medullary subdural nodules of the lumbar spine, neurogenic tumors, meningioma. The patient received sacrosacral sinus excision.

Pathology (lumbosacral skin and subcutaneous tissue) revealed cuticle cyst in subcutaneous tissue. After the surgery, the patient received postoperative continuous lateral ventricle drainage for 21days. And amikacin (10mg/kg) with dexamethasone $(0.5 \mathrm{mg})$ were given by lateral ventricle for 7 days. The brain MRI showed 
that the hydrocephalus was alleviated. 21 days after the surgery, the patient received lateral ventriculo ventriculoperitoneal shunt. After 3 days anti-infection with ceftazidime $(120 \mathrm{mg} / \mathrm{kg}$. d), the patient was discharged. One month after discharge, the child had recurrent fever again, with 2 times' convulsions. New symptoms appeared, including astasia, urine incontinence and constipation. Lumbar vertebra MRI showed that intraspinal tumor and syringomyelia at the lumbar 3-4 level. The patient received intraspinal occupying resection and neurolysis. Intraspinal occupying culture revealed: Escherichia coli. Pathology suggested: mature teratoma. Antibiotics were used according to drug sensitivity test. After the treatment, the child recovered well and was discharged from the hospital. The patient was followed up with no recurrence.

\section{Systematic Review of the Literature}

Using dermal sinus and bacterial meningitis as key words, we searched databases including PubMed, Embase and Chinese databases such as CNKI, VIP, and Wanfang database from the beginning of the database to November 2017. We have 22 Chinese articles and 77 English articles. Repetitive studies, non-Chinese or English literature that cannot be analysis from the cases were excluded; we got 68 cases bacterial meningitis caused by sacral sinus. Most of the patients, age from neonate [1] to adult [2], complained of fever, headache, vomiting and convulsions. Few people had back pain, lower limb pain and limping as initial symptoms. And some patients didn't have typical symptoms while headache and vomit were transient.

Some patients were admitted in hospital without any symptoms and signs except fever. That may be related to repeated infection of low toxicity bacteria, opportunistic pathogens and early application of antibiotics. Some patients who had obscure onset and prolonged course were misdiagnosed as tuberculosis meningitis due to lack of systemic toxic symptoms and acute inflammatory reaction. In cases reported on the dermal sinus, there were 13 cases of occipital, 1 case of neck, 5 cases of chest and 18 cases in lumbosacral region. In those cases with pathogens isolated from blood or cerebrospinal fluid samples, 2 samples were proteus, 3 samples were Staphylococcus aureus, 3 samples were Escherichia coli, 2 samples were group B Streptococcus and 1 sample was Klebsiella pneumoniae. Other pathogens include Pseudomonas aeruginosa, anaerobes, Staphylococcus aureus and Escherichia coli [3]. Most of the pathogenic bacteria were derived from the normal colonization on the skin. Morgenstern believed that different bacterial meningitis caused by anatomic abnormalities (including the sinus), can provide some useful suggestion in the location of the abnormalities [4]. However, the results of the pathogen literature analysis showed that the implications were very limited.

\section{Discussion}

It was quite hard to diagnose and treat congenital dermal sinus if the patients only had clinical manifestations of bacterial meningitis, while with insidious sinus orifice and without local infection. It may be hard for the patients having dermal sinus with spinal endothelial cysts or epithelial cysts to have sinus and cysts completely removed because of tight adhesion with the spinal cord or nerve root. In this case, although the child had definite infectious pathogen and received enough dosage of effective antibiotics, meningitis was still recurring. He was treated by surgical excision of the dermal sinus in the first surgery. But the cyst in the spinal canal was not thoroughly removed. So the patient had re-infection of the same pathogen. In the second surgery, after the cyst in the spinal canal was thoroughly removed, no recurrence was found. So early diagnosis and surgical treatment is appreciated. Escherichia coli are one of the common pathogen in infant's bacterial meningitis. It is necessary to find out the reason why the symptoms recurring after receiving appropriate antibiotics treatment. Immune deficiency, congenital anatomic abnormality, trauma and operation are all important factors of recurrent meningitis. From the experience of this case, there was something we should pay attention to:

a. Inquire the patient history detailed;

b. Systematic physical examination, especially the midline craniospinal axis;

c. Proper imaging examination besides brain MRI.

Spinal MRI is the best imaging method for the diagnosis of intraspinal cysts, which can indicate the position of the cyst, and provide a reliable basis for clinical diagnosis and operation. In the treatment, we should actively search for the infection pathogens, select sensitive drugs according to the results of drug sensitivity experiments combined with clinical efficacy, and combined treatment should be taken into consideration when necessary. Early surgical treatment should be considered while controlling infection. In this case, the prognosis of the patient was good after thoroughly surgical removal of the sinus and spinal canal cyst which were the primary infective focus. But for children with complications of intracranial and intraspinal abscess, which caused severe neurological dysfunction, without timely surgery, the prognosis was poor. Delayed diagnosis is mainly due to the doctor' unawareness of this type of anatomic abnormality. The importance of early diagnosis, proper examinations, surgical treatment and close follow up in patients with recurrent meningitis is stressed.

\section{References}

1. Shimizu M, Araki R, Niida Y, Nakada M, Yachie A, et al. (2009) Mollaret meningitis associated with occipital dermal sinus[J]. J Pediatr 155(5): 757.

2. Reimer CM (2001) Dermal sinus presenting as group B streptococcal meningitis [J]. Archives of Neurology 58(5): 820.

3. Kanev PM, Salazar JC (2010) Unusual CNS infection from a subtorcular dermal sinus [J]. Acta Paediatrica, International Journal of Paediatrics 99(4): 627-629.

4. Morgenstern IA, Bach FA, Martinez S, Martin NA, Vazquez ME, et al. (2014) Recurrent meningitis due to anatomical defects: The bacteria indicates its origin [J]. An Pediatr (Barc) 82(6): 388-396. 


$\begin{array}{ll}\text { BIOMEDICAL } & \text { Assets of Publishing with us } \\ \text { RESEARCHES } & \text { Global archiving of articles }\end{array}$

